\title{
Minimum Intersymbol Interference Methods for Time Domain Equalizer Design
}

\author{
Ming Ding ${ }^{1}$, Brian L. Evans ${ }^{1}$, Richard K. Martin², and C. Richard Johnson, Jr., ${ }^{2}$ \\ (1) Dept. of Electrical and Comp. Eng., \\ The University of Texas at Austin, Austin, TX 78712 USA \\ email: \{ming,bevans\}@ece.utexas.edu \\ (2) School of Electrical and Computer Engineering \\ Cornell University, Ithaca, NY, 14853 USA \\ email: \{frodo,johnson\}@ece.cornell.edu
}

\begin{abstract}
During initialization, discrete multitone receivers train a time domain equalizer (TEQ) to shorten the channel impulse response to a preset length, $\nu+1$. Arslan, Kiaei, and Evans report a Minimum Intersymbol Interference (Min-ISI) method for TEQ design. Min-ISI TEQs give the highest bit rates among single-FIR TEQs amenable to real-time implementation on programmable fixed-point digital signal processors (DSPs). The Min-ISI method, however, has several disadvantages: (1) sensitivity to transmission delay, (2) inability to design TEQs longer than $\nu+1$ taps, and (3) sensitivity to the fixed-point computation in the Cholesky decomposition. In this paper, we develop an alternate Min-ISI cost function, from which we derive (1) a fast search method for the optimal transmission delay, (2) extensions to design arbitrary-length Min-ISI TEQs, and (3) an iterative Min-ISI method. The iterative Min-ISI method avoids Cholesky decomposition, designs arbitrary length TEQs, and achieves the bit rate performance of the original Min-ISI method.
\end{abstract}

\section{INTRODUCTION}

Discrete multitone (DMT) modulation is extensively used in broadband wireline communication systems, such as asymmetric digital subscriber line (ADSL) and very-high speed DSL systems (VDSL). A reason for DMT's popularity is the tradeoff in implementation complexity vs. achievable bit rates. A key implementation efficiency comes from using the fast Fourier transform (FFT) for the multicarrier modulation and demodulation.

An ADSL frame consists of a concatenation of a cyclic prefix and a symbol of $N$ samples, where the cyclic prefix is a copy of the last $\nu$ samples of the symbol. The receiver performs time-domain equalization and frequency-domain equalization. Time-domain equalization makes the effective discretized channel impulse response be no longer than $\nu+1$ samples. The channel shortening ideally converts a linear convolution into a circular convolution.

The conventional DMT equalizer structure consists of a cascade of a single-FIR time-domain equalizer (TEQ), FFT, and single-tap frequency-domain equalizer (FEQ). DMT TEQ design methods optimize different criteria to compute the FIR coefficients based on training data. Minimum mean squared error (MMSE) design, which has its

M. Ding and B. Evans were supported by The State of Texas Advanced Technology Program under project 003658-0614-2001 roots in [1], was revived in the early stages of ADSL research [2], [3]. MMSE TEQ design minimizes the mean square error between the output of the physical path consisting of the channel and FIR filter and the output of a virtual path consisting of a transmission delay $\Delta$ and a target impulse response (TIR). An iterative implementation of the MMSE TEQ design method [4] shipped in many of the ADSL modems in the late 1990s. Drawbacks of the MMSE TEQ method include sensitivity to the transmission delay parameter and nulling of subcarriers [5]. Bit rate performance varies widely with TEQ length. At some point, longer TEQs start killing subcarriers [6].

The maximum shortening SNR (MSSNR) [7] method attempts to minimize intersymbol interference (ISI) in the time domain. The MSSNR method maximizes the ratio of the energy of the effective channel impulse response inside a target window of $\nu+1$ samples to that outside the target window. Alternate objective functions include maximizing the ratio of the energy inside the target window to the total energy [9], [16], and minimizing (maximizing) the energy outside (inside) the target window while holding the energy inside (outside) the target window fixed. Finitelength MSSNR TEQs are approximately symmetric [10]. A blind, adaptive MSSNR algorithm is reported in [11]. Drawbacks of the MSSNR TEQ method include sensitivity to the transmission delay parameter and lack of control of where the ISI resides in the frequency domain. Again, bit rate performance varies widely with TEQ length. At some point, longer TEQs start killing subcarriers [6].

The Minimum-ISI (Min-ISI) method generalizes the MSSNR method by weighting the ISI in the frequency domain [12]. Frequency-weighted ISI is an approximation of achievable bit rate. Min-ISI TEQs give the highest bit rates among single-FIR TEQs amenable to real-time implementation on programmable fixed-point DSPs. Drawbacks include: (1) sensitivity to transmission delay, (2) inability to design TEQs longer than $\nu+1$ taps, and (3) sensitivity to the fixed-point computation in the Cholesky decomposition. On fixed-point DSPs with 16-bit multipliers, e.g. TI TMS320C6200 and Motorola 56300, the Cholesky decomposition may become unstable for TEQs longer than 
15 taps.

At least three alternate equalizer structures have been proposed. The dual-FIR TEQ [13] uses a standard singleFIR TEQ design algorithm to achieve good performance over the entire transmission bandwidth, and uses a secondFIR TEQ design algorithm to achieve better performance over a subset of subcarriers. A second alternate structure is the per-tone equalizer [14]. The per-tone equalizer essentially moves the single-FIR TEQ into the FEQ, which makes the FEQ become a linear combiner for each subcarrier. For data transmission, the per tone equalizer requires several times more memory but slightly lower computational complexity than the conventional equalizer. For training, however, the implementation complexity can increase by up to a factor equal to the number of subcarriers, $N / 2$, over the conventional equalizer. A third alternate structure is a filter bank TEQ, in which a different FIR TEQ is designed for each tone. The FFT becomes a bank of Goertzel filters [15]. As reported in [15], the filter bank TEQ method has nine times the computational complexity of the per-tone equalizer for ADSL data transmission.

In this paper, we reformulate the Min-ISI cost function to enable it to be applied to the design of arbitrary length TEQs. The new cost function yields a significant reduction in computational complexity in searching for the optimal transmission delay. To reduce the number of multiplications, we introduce quantized frequency-domain ISI weighting functions. We derive an iterative version of the Min-ISI method, based on the new cost function. The iterative method does not require any Cholesky decompositions and is well suited for fixed-point implementation.

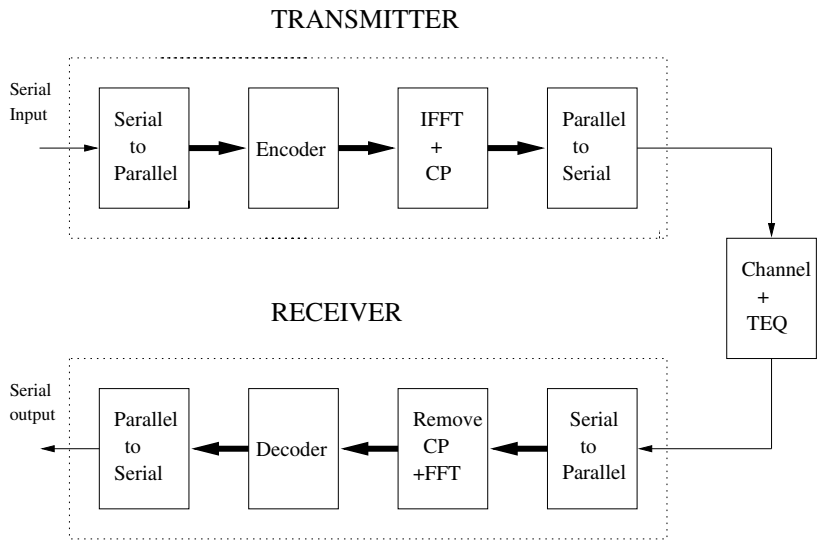

Fig. 1. Block diagram of a DMT transceiver

\section{BACKGROUND}

\section{A. System Model}

Fig. 1 depicts a block diagram of a DMT transceiver. In the transmitter, the data sequence is partitioned into a number of parallel streams. Each stream of data is modulated via a particular subcarrier. The modulated subcarriers are summed to obtain the transmit signal. The use of the discrete Fourier transform (DFT)in DMT allows an efficient realization of the subcarrier modulators in a parallel processing structure that benefits from the computational efficiency of the FFT. A similar DFT-based structure is used for efficient realization of the subcarrier demodulators in the receiver part of the DMT modem. As mentioned in Section I, ISI could ideally be removed by cyclically extending the output of the inverse FFT modulator so that the input sequence looks periodic to the channel. The length of the cyclic prefix should be at least equal to the duration of the channel impulse response, $L_{h}$, minus one. However, we note that the addition of the cyclic prefix reduces the throughput of the channel as it carries redundant data. To minimize this reduction of throughput, a TEQ is applied to reduce the overall duration of the system (channel plus equalizer) impulse response to a predefined length.

In explaining the function of the TEQ, let $t$ indicate the DMT symbol index and $n=0,1, \ldots, N+\nu-1$ indicate the samples within the given symbol plus the CP length $\nu$. The TEQ output is given as

$$
z_{t}(n)=\sum_{\tau=0}^{L_{w}-1} w(\tau) r_{t}(n-\tau)
$$

where $w(\tau)$ denotes the $\tau$ th coefficient of the length $L_{w}$ TEQ and $r_{t}(\cdot)$ is the received sequence.

Next, we remove the samples corresponding to the CP of the TEQ output and transform the result to frequency domain by means of a FFT. To recover the transmitted data, a one-tap FEQ is applied for each tone of the FFT output to undo the attenuation of the shortened channel.

\section{B. Review of the Min-ISI Method}

The Min-ISI method attempts to optimize an approximation of the achievable bit rate [12]. The Min-ISI method decomposes the equalized channel output $h(n) * w(n) * x(n)$ into the desired part and ISI corrupted part by applying on a window function.

$$
g(n)= \begin{cases}1 & \text { if } \Delta \leq n \leq \Delta+\nu \\ 0 & \text { elsewhere }\end{cases}
$$

where $\Delta$ is the transmission delay. The TEQ output becomes

$$
\begin{aligned}
y(n)= & h(n) * w(n) * x(n)+w(n) * \nu(n) \\
= & h^{\text {signal }}(n) * x(n)+h^{I S I}(n) * x(n) \\
& +w(n) * \nu(n)
\end{aligned}
$$

Define

$$
\begin{aligned}
& \mathbf{w}=\left[\begin{array}{llll}
w(0) & w(1) & \ldots & w\left(L_{w}-1\right)
\end{array}\right]^{T} \\
& \mathbf{G}=\operatorname{diag}\left[\begin{array}{llll}
g(0) & g(1) & \ldots & g(N-1)
\end{array}\right]^{T} \\
& \mathbf{D}=\mathbf{I}-\mathbf{G} \\
& \mathbf{H}=\left[\begin{array}{cccc}
h(0) & h(-1) & \ldots & h\left(-\left(L_{w}-1\right)\right) \\
h(1) & h(0) & \ldots & h\left(-\left(L_{w}-2\right)\right) \\
\vdots & \vdots & \ddots & \vdots \\
h(N-1) & h(N-2) & \ldots & h\left(N-L_{w}\right)
\end{array}\right] \\
& \mathbf{q}_{i}=\left[\begin{array}{llll}
1 & e^{j 2 \pi i / N} & \ldots & e^{j 2 \pi i(N-1) / N}
\end{array}\right]^{T}
\end{aligned}
$$


The Min-ISI cost function can be written as [12]

$$
\begin{aligned}
J(\mathbf{w}) & =\frac{\mathbf{w}^{T} \mathbf{H}^{T} \mathbf{D}^{T} \sum_{i}\left(\mathbf{q}_{i} \frac{S_{x, i}}{S_{n, i}} \mathbf{q}_{i}^{H}\right) \mathbf{D H w}}{\mathbf{w}^{T} \mathbf{H}^{T} \mathbf{G}^{T} \mathbf{G H} \mathbf{w}} \\
& =\frac{\mathbf{w}^{T} \mathbf{X} \mathbf{w}}{\mathbf{w}^{T} \mathbf{Y} \mathbf{w}}
\end{aligned}
$$

where $S_{x, i}$ and $S_{n, i}$ are the transmitted signal power and channel noise power for $i$ th tone, respectively.

\section{Min-ISI FOR ARBITRARY LENGTH TEQ}

One can convert the Min-ISI TEQ design problem to a constrained minimization problem by setting $\mathbf{w}^{T} \mathbf{Y} \mathbf{w}=1$ :

$$
\min _{\mathbf{w}}\left(\mathbf{w}^{T} \mathbf{X w}\right) \quad \text { subject to } \mathbf{w}^{T} \mathbf{Y} \mathbf{w}=1
$$

The solution is obtained by solving the generalized eigenvector problem

$$
\mathbf{X} \mathbf{w}=\tilde{\lambda} \mathbf{Y} \mathbf{w}
$$

where $\tilde{\lambda}$ is the smallest generalized eigenvalue of the matrix pencil $(\mathbf{X}, \mathbf{Y})$.

When $L_{w}>\nu+1$, a case mostly happens in upstream transmission, $\mathbf{G H}$ is rank deficient and $\mathbf{Y}$ will not be invertible. We cannot apply the method in [7] to solve the problem. Thus, the original formulation of Min-ISI is not suitable for design of arbitrary length TEQs.

Instead, we follow the approach in [16], which solves

$$
\mathbf{Y w}=\lambda \mathbf{X w}
$$

where $\lambda=1 / \tilde{\lambda}$ is the largest eigenvalue of the matrix pencil $(\mathbf{Y}, \mathbf{X})$. It is observed that in practice $\mathbf{X}$ is always invertible for all channels longer than the $\mathrm{CP}$. However, the dependence between $\mathbf{X}$ and the windowing function $\mathbf{G}$ introduces additional computation.

The original formulation minimizes the ratio of a weighted sum of the ISI power over the sum of the desired signal power within a target window. The target window starts from a given delay $\Delta$ and ends at $\Delta+\nu$. To find the optimum $\Delta$, we would need to search over all possible $\Delta$ values. For each delay, we use the general eigen-approach to find the TEQ that minimizes the cost function. Since $\mathbf{Y}$ is a function of $\Delta$, we need as many number of Cholesky decompositions as the number of searched $\Delta$ values. The alternative approach in (8) faces the same problem since $\mathbf{X}$ is also a function of delay $\Delta$.

However, we reformulate the Min-ISI cost function not only to make it suitable for arbitrary length TEQ design, but also to avoid need for multiple Cholesky decompositions. In a similar fashion to recently proposed delay spread equalizers [8], [9], we change the definition of matrix $\mathbf{Y}$ in (5) from $\mathbf{H}^{T} \mathbf{G}^{T} \mathbf{G H}$ to $\mathbf{H}^{T} \mathbf{H}$. With this change, we aim to minimize the ratio of the weighted sum of ISI power over the total signal power instead of the windowed signal power. Mathematically we reach the same optimum as the original one but with fewer computations when searching for optimal delay. The new $\mathbf{Y}$ is a positive definite matrix [6] and a Cholesky decomposition $\mathbf{Y}=\mathbf{L L}^{T}$ can be performed. The modified min-ISI has a cost function as

$$
J(\mathbf{w})=\frac{\mathbf{w}^{T} \mathbf{X} \mathbf{w}}{\mathbf{w}^{T} \mathbf{L} \mathbf{L}^{T} \mathbf{w}}
$$

We notice $\mathbf{L}$ here does not depend on $\Delta$, so only one Cholesky decomposition is needed for the modified MinISI method. Once $\mathbf{L}$ is available, it can be used for any delays. Also we noticed $\mathbf{Y}$ is always invertible, this new formulation can be applied to the design of TEQ with any number of taps.

The formulation of $\mathbf{Y}$ takes more computation than the original one, but it is negligible if compared to the reductions of multiple Cholesky decompositions when searching for the optimum delay.

A rough analysis of computation complexity is as follows. All possible transmission delays range from 0 to $N-\nu$, which is 481 in an ADSL system. For a nonToeplitz $L_{w} \times L_{w}$ matrix $\mathbf{Y}$, the Cholesky decomposition can be computed in $\left(L_{w}\right)^{3} / 3$ floating operations [17]. Thus, for a 17-tap TEQ, the savings in computations is $786080-16320=769760$ operations, where the second item to be subtracted is the additional operations for calculation of the new $\mathbf{Y}$.

\section{Quantized ISI Frequency Weighting}

In the original Min-ISI cost function in (5), the weighting $\sum_{i}\left(\mathbf{q}_{i} \frac{S_{x, i}}{S_{n, i}} \mathbf{q}_{i}^{H}\right)$ pushes the ISI power into the subchannels with lower SNR. The MSSNR method, however, treats ISI in low and high SNR subchannels equally. Hence, Min-ISI outperforms MSSNR in terms of achievable bit rate.

In the Min-ISI method, we need multiplication operations to implement the weighting function. To reduce computational complexity, we quantize the SNR weighting. First, we threshold the SNR calculation to compute "onoff" weighting. We compare the $\frac{S_{x, i}}{S_{n, i}}$ to a preset threshold $T_{i}$, and set the weighting to 1 if the condition is true and 0 otherwise. That is, an "off" weighting of 0 means that the noise power is too strong in that subcarrier, and we do not care whether or not ISI is minimized there. In the case of the G.DMT ADSL standard, a meaningful threshold is the subcarrier SNR value that results in a two-bit allocation.

When the transmission has a flat spectrum, as in the case of transmission of the training sequences in the G.DMT ADSL standard, the comparison would reduce to a simple subtraction operation, $S_{n, i}-S_{n, p r e s e t}$. For the on-off case, the preset value $S_{n, p r e s e t}$ may be calculated offline by

$$
S_{n, \text { preset }}=\frac{S_{x}}{3 \times 10^{\left(\Gamma_{g a p} / 10\right)}}
$$

where $S_{x}$ is the common value of signal power for all subcarriers and $\Gamma_{g a p}$ is SNR gap for achieving Shannon channel capacity.

This digitized "on-off" weighting drops the subcarriers which cannot carry at least 2 bits and saves multiplication operations. Thus the implementation complexity is reduced. We notice that MSSNR is a special case of this onoff weighting which turns on all of the subcarriers. Min-ISI 
with quantized weights treats all of the subcarriers above the threshold equally, so somehow the ability to emphasize on the subcarriers with highest SNR is limited when compared to the original Min-ISI. But it works well in the case of downstream Frequency Division Multiplexing (FDM) ADSL, in which 40 or so subcarriers with the highest SNR are reserved for upstream data transmission. Also, we observe this on-off weighting method works well when severe crosstalk, which has irregular noise power spectral density, is present. Sometimes on-off Min-ISI has better performance than both the MSSNR and Min-ISI.

The on-off weighting can be extended to multiple levels if we use multiple thresholds and assign different weighting values. For G.DMT ADSL, each subcarrier can support 0-15 bits. (There is provision to support one bit on a subcarrier, although this provision is rarely implemented.) We can put four thresholds and assign weighting values as 0 , $1,2^{1}, 2^{2}$. Quantization to 4 levels can be performed with two comparisons. The weighting can be implemented as shifting, which is still a computational savings over multiplication.

\section{Proposed Iterative Min-ISI algorithm}

Considering all the refinements mentioned in previous sections, we propose an iterative algorithm here that can be easily applied once the channel estimates and the frequency weighting functions are available. Hereafter we denote the weighting function for subcarrier $i$ as $\alpha_{i}$. In the original Min-ISI, $\alpha_{i}=\frac{S x_{i}}{S n_{i}}$, which can be estimated from Reverb (a periodic sequence, which therefore does not need a TEQ) transmitted in the early stage of receiver initialization. Reasonable digitization could be performed in the proposed algorithm.

The cost function can be written as

$$
\begin{aligned}
J\left(\mathbf{w}_{k}\right) & =\mathbf{w}_{k}^{T} \mathbf{H}^{T} \mathbf{D}^{T} \sum_{i}\left(\mathbf{q}_{i} \alpha_{i} \mathbf{q}_{i}^{H}\right) \mathbf{D H} \mathbf{w}_{k} \\
& =\mathbf{w}_{k}^{T} \mathbf{X} \mathbf{w}_{k}
\end{aligned}
$$

where $\mathbf{X}$ only differs from the definition in (5) in the weights $\alpha_{i}$.

The performance surface is quadratic and the method of steepest descent is readily implemented. The gradient $\nabla_{k}$ is obtained by

$$
\begin{aligned}
\nabla_{k} & =\left.\frac{\partial J}{\partial \mathbf{w}}\right|_{\mathbf{w}=\mathbf{w}_{k}}=\frac{\left(\partial\left(\mathbf{w}_{k}^{T} \mathbf{X} \mathbf{w}_{k}\right)\right.}{\partial \mathbf{w}_{k}} \\
& =\left(\mathbf{X}+\mathbf{X}^{T}\right) \mathbf{w}_{k}
\end{aligned}
$$

The iterative update of TEQ is

$$
\mathbf{w}_{k+1}=\mathbf{w}_{k}+\mu\left(-\nabla_{k}\right)
$$

where $\mu$ is the step size which regulates the speed and stability of adaptation. The constraint $\mathbf{w}^{T} \mathbf{Y} \mathbf{w}$ should also be included, which can be implemented by renormalizing $\mathbf{w}$ after each iteration. Fig. 2 presents the full algorithm.
1. Obtain the weighting values $\alpha_{i}=\frac{S x_{i}}{S n_{i}}$.

2. Pre-compute $L_{w} \times L_{w}$ Hermitian matrix $\mathbf{X}=\mathbf{H}^{T} \mathbf{D}^{T} \sum_{i}\left(\mathbf{q}_{i} \alpha_{i} \mathbf{q}_{i}^{H}\right) \mathbf{D H}$ and $\hat{\mathbf{X}}=\mathbf{X}+$ $\mathbf{X}^{T}$, where $\mathbf{H}, \mathbf{D}$, and $\mathbf{q}_{i}$ are defined in (4).

3. Start with non-zero initial guess $\mathbf{w}_{\mathbf{0}}$

4. $\nabla_{k}=\hat{\mathbf{X}} \mathbf{w}_{k}$

5. $\hat{\mathbf{w}}_{k+1}=\mathbf{w}_{k}+\mu\left(-\nabla_{k}\right)$

6. $\mathbf{w}_{k+1}=\frac{\hat{\mathbf{w}}_{k+1}}{\sqrt{\hat{\mathbf{w}}_{k+1}^{T} \mathbf{Y} \hat{\mathbf{w}}_{k+1}}}$

Fig. 2. Proposed iterative Min-ISI TEQ design method

\section{Simulations}

To evaluate the performance of the proposed iterative min-ISI design method and also to compare it with the original min-ISI method and other major algorithms, we present the results of a number of TEQs which we have designed for 8 typical CSA loops that can be obtained from [18]. The DMT setup that we consider is the downstream of an FDM ADSL transceiver. Accordingly, the IFFT and FFT lengths are 512 and a cyclic prefix length of 32 is assumed. The signal power spectral density at the transmitter output is set equal to $-40 \mathrm{dBm} / \mathrm{Hz}$. Channel noise is modeled as an additive white Gaussian noise (AWGN) with $-140 \mathrm{dBm} / \mathrm{Hz}$ power density.
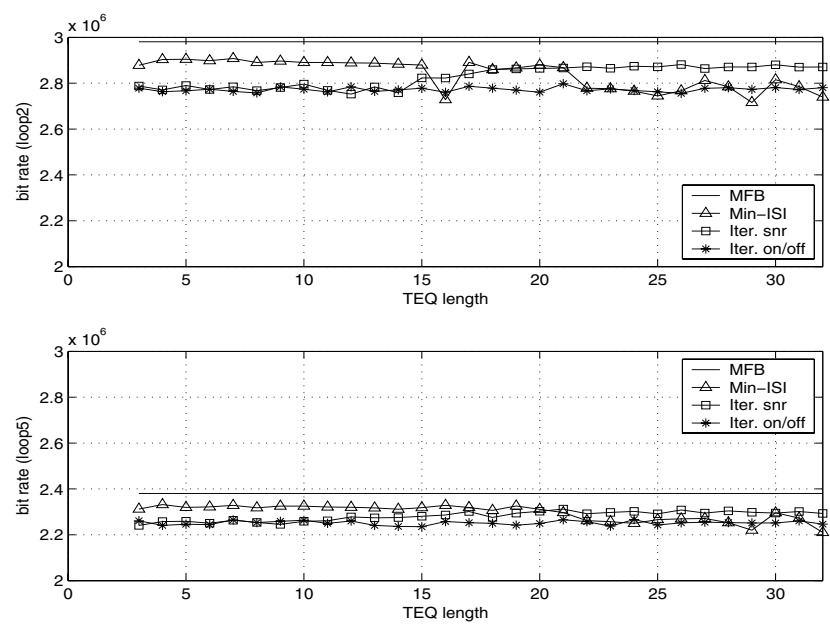

Fig. 3. Achievable bit rate vs. TEQ length for CSA loops 2 and 5. Coding gain is $5 \mathrm{~dB}$, margin is $6 \mathrm{~dB}$, input power is -40 $\mathrm{dBm} / \mathrm{Hz}$, AWGN power $-140 \mathrm{dBm} / \mathrm{Hz}$, NEXT noise is from 24 HDSL disturbers. Equalizer is trained by the Min-ISI and Iterative Min-ISI, with SNR weighting and on-off weighting.

Table 1 presents a summary of the results that we obtained for a 5-tap TEQ design using MMSE, MSSNR, the original Min-ISI, proposed iterative Min-ISI. For the iterative method, weighting function $\alpha_{i}=\frac{S x_{i}}{S n_{i}}$ is chosen. The initial guess is $\mathbf{w}_{0}=\left[\begin{array}{ll}1 & \mathbf{0}_{\left(L_{w}-1\right) \times 1}\end{array}\right]^{T}$, the step size is set to $10^{-7}$, and the number of iterations is 30 . The optimum delay $\Delta$ is obtained by searching from 5 to 40 . 


\section{TABLE I}

Achievable bit Rate (IN MbPs) For 8 CSA loops USING FDM-ADSL TRANSMISSION FOR FOUR TEQ DESIGN METHODS COMPARED AGAinst THE MATCHED FILTER BOUND (MFB). TEQ LENGTH IS 5 TAPS, CODING GAIN IS 5 DB, MARGin is 6 DB, INPUT POWER Is $-40 \mathrm{DBM} / \mathrm{Hz}$, AND AWGN POWER IS $-140 \mathrm{DBM} / \mathrm{Hz}$.

\begin{tabular}{c|c|c|c|c|c}
\hline & \multicolumn{4}{|c|}{ achievable percentage of MFB bit rate } & bit rate \\
\hline \hline loop & MMSE & MSSNR & Min-ISI & Iter. & MFB \\
\hline 1 & $43 \%$ & $96 \%$ & $98 \%$ & $97 \%$ & 9.801 \\
2 & $33 \%$ & $95 \%$ & $98 \%$ & $98 \%$ & 11.001 \\
3 & $41 \%$ & $96 \%$ & $99 \%$ & $98 \%$ & 9.407 \\
4 & $38 \%$ & $97 \%$ & $98 \%$ & $97 \%$ & 9.427 \\
5 & $34 \%$ & $97 \%$ & $98 \%$ & $98 \%$ & 9.963 \\
6 & $43 \%$ & $97 \%$ & $98 \%$ & $98 \%$ & 9.132 \\
7 & $33 \%$ & $98 \%$ & $99 \%$ & $99 \%$ & 8.975 \\
8 & $29 \%$ & $98 \%$ & $99 \%$ & $99 \%$ & 7.957 \\
\hline \hline
\end{tabular}

Bit allocation on different subcarriers is calculated by

$$
b_{i}=\log _{2}\left(1+\frac{\mathrm{SNR}_{i}}{\Gamma_{\text {sim }}}\right)
$$

where $i$ varies over all data carrying subcarriers, $\mathrm{SNR}_{i}$ is the SNR at the $i$ th subcarrier, and

$$
\Gamma_{\text {sim }}(\text { in } \mathrm{dB})=\Gamma_{\text {gap }}+\text { system margin }- \text { coding gain }
$$

where $\Gamma_{\text {gap }}=9.8 \mathrm{~dB}$ corresponds to $10^{-7}$ bit error rate, system margin is $6 \mathrm{~dB}$, and coding gain is $5 \mathrm{~dB}$. The total number of bits per DMT symbol is $\sum_{i} b_{i}$.

The results suggest that the iterative Min-ISI method eventually achieves the same bit rates as the original MinISI method in an FDM-ADSL system. Both methods perform closer to the Matched Filter Bound (MFB). For the original Min-ISI method, the gap from the MFB is narrower for FDM-ADSL than the echo-cancelled ADSL results in [12]. The MSSNR method shows similar improvement. MMSE performance, however, is lower than its performance for echo-cancelled ADSL. That is because MMSE puts more emphasis on subchannels with highest SNR, which are not used in FDM-ADSL downstream.

We also test the proposed iterative method with on-off weighting when severe crosstalk is present. Channel noise is modeled as near-end-cross-talk (NEXT) from 24 high speed DSL (HDSL) disturbers plus additive white Gaussian noise (AWGN) with $-140 \mathrm{dBm} / \mathrm{Hz}$ power density. Fig. 3 shows the bit rate performance for CSA loop 2 and 5 vs. TEQ length. The results of original and iterative MinISI methods with non-quantized weighting functions are also provided for comparison. All the three Min-ISI methods perform quite close to the MFB when severe crosstalk is present. There is no noticeable difference between iterative implementation with on-off weighting and original weighting. All the three Min-ISI methods have "flat" bit rate performance vs. various TEQ length. It confirms that we can effectively shorten a downstream channel with very short TEQs using Min-ISI methods.

\section{ViI. Conclusions}

This paper derives a new cost function that measures ISI at the FFT output. We use the new cost function to design Min-ISI TEQs longer than the cyclic prefix length. We also use the new cost function to reduce the number of Cholesky decompositions when searching for the optimal transmission delay to one. We introduce quantized ISI frequency weightings to reduce computational complexity.

Based on the new cost function, we derive an iterative Min-ISI method. The iterative Min-ISI method avoids Cholesky decomposition entirely, designs arbitrary length TEQs, and achieves the bit rate performance of the original Min-ISI method. The iterative Min-ISI method is amenable to implementation on fixed-point programmable digital signal processors.

\section{REFERENCES}

[1] D. D. Falconer and F. R. Magee, "Adaptive Channel Memory Truncation for Maximum Likelihood Sequence Estimation," Bell Sys. Tech. Journal, pp. 1541-1562, Nov. 1973.

[2] J. S. Chow, J. M. Cioffi, and J. A. C. Bingham, "Equalizer Training Algorithms for Multicarrier Modulation Systems," in Proc. IEEE Int. Conf. on Comm., May 1993, pp. 761-765.

[3] N. Al-Dhahir and J. M. Cioffi, "Efficiently Computed ReducedParameter Input-Aided MMSE Equalizers for ML Detection: A Unified Approach," IEEE Trans. on Info. Theory, vol. 42, no. 3, pp. 903-915, May 1996.

[4] J. S. Chow, J. C. Tu, and J. M. Cioffi, "A Discrete Multitone Transceiver System for HDSL Applications," IEEE J. Selected Areas Comm., vol. 9, no. 6, pp. 895-907, Aug. 1991.

[5] B. Farhang-Boroujeny and M. Ding, "Design Methods for TimeDomain Equalizers in DMT Transceivers," IEEE Trans. on Comm., vol. 49, no. 3, pp. 554-562, Mar. 2001.

[6] R. K. Martin, C. R. Johnson Jr., M. Ding, and B. L. Evans, "Infinite Length Results for Channel Shortening Equalizers," in Proc. IEEE Signal Proc. Adv. in Wireless Comm., June 2003.

[7] P. J. W. Melsa, R. C. Younce, and C. E. Rohrs, "Impulse Response Shortening for Discrete Multitone Transceivers," IEEE Trans. on Comm., vol. 44, pp. 1662-1672, Dec. 1996.

[8] R. Schur and J. Speidel, "An Efficient Equalization Method to Minimize Delay Spread in OFDM/DMT Systems," in Proc. IEEE Int. Conf. on Comm., vol. 1, pp. 1-5, June 2001.

[9] A. Tkacenko and P. P. Vaidyanathan, "Noise optimal eigenfilter design of time domain equalizers for DMT systems," in Proc. IEEE Int. Conf. on Comm., vol. 1, pp. 54-58, Apr. 2002.

[10] R. K. Martin, C. R. Johnson Jr., M. Ding, and B. L. Evans, "Exploiting Symmetry in Channel Shortening Equalizers," in IEEE Int. Conf. on Sig. Proc., vol. v, pp. 97-100, Apr. 2003.

[11] R. K. Martin, J. Balakrishnan, W. A. Sethares, and Jr. C. R. Johnson, "A Blind, Adaptive TEQ for Multicarrier Systems," IEEE Signal Proc. Let., vol. 9, pp. 341-343, Nov. 2002.

[12] G. Arslan, B. L. Evans, and S. Kiaei, "Equalization for Discrete Multitone Receivers To Maximize Bit Rate," IEEE Trans. on Signal Processing, vol. 49, no. 12, pp. 3123-3135, Dec. 2001.

[13] M. Ding, A. J. Redfern, and B. L. Evans, "A Dual-path TEQ Structure For DMT-ADSL Systems," in IEEE Int. Conf. on Sig. Proc., May 2002, pp. 2573-2576.

[14] K. Van Acker, G. Leus, M. Moonen, O. van de Wiel, and T. Pollet, "Per Tone Equalization for DMT-Based Systems," IEEE Trans. on Comm., vol. 49, no. 1, pp. 109-119, Jan. 2001.

[15] M. Milosevic, L. F. C. Pessoa, B. L. Evans, and R. Baldick, "DMT Bit Rate Maximization With Optimal Time Domain Equalizer Filter Bank Architecture," in Proc. IEEE Asilomar Conf. on Sig., Sys., and Comp., vol. 1, pp. 377-382, Nov. 2002.

[16] C. Yin and G. Yue, "Optimal Impulse Response Shortening for Discrete Multitone Transceivers," Elect. Let., vol. 34, pp. 35-36, Jan. 1998.

[17] T. K. Moon and W. C. Stirling, Mathematical Methods and Algorithms for Signal Processing, Prentice Hall, 2000.

[18] G. Arslan, M. Ding, B. Lu, M. Milosevic, Z. Shen, and B. L. Evans, "TEQ design toolbox," The University of Texas at Austin, www.ece.utexas.edu/ bevans/projects/adsl/dmtteq. 